V. Ueber den Einflufs der Gebirge auf die Winde des angränzenden Flachlandes; ion $W$. C. Wittwer.

Seit langer Zeit keunt man die Thatsache, dafs der Wind sich an manchen Orten während der verschiedenen Tageszeiten ändert; es brauchen hier nur die allgemein bekanten Land. und Seewinde angeführt zu werden. Aber auch im Innern der Continente fern von jeder gröfseren Wasserfläche begegnet man solchen Erscheinungen und besonders siud es die Gebirgsgegenden, die sich in dieser Beziebung auszeichnen. Manche Bergwinde, wie z. B. die Born, sind sogar unit eigenen Namen bedacht und viele Bewohner der Gebirge, deren Beschäfligung wie die der Jïger auf Berücksichtigung der Winde hinweist, kennen sehr gut die Luftströmungen, auf die zu gegebenen Tageszeiten an bestimınten Stellen zu rechnen ist.

Wenu nun hier durchaus nicht vou Bekanutmachung einer neuen Thatsache die Rede seyn. kanu, so dürfle es doch nicht überflüssig seyn, die Normen, nach denen sich die Winde des dem Gebirge nahen Flachlaudes regeln, genauerer Untersuchung zu unterzichen.

Der Einflufs, den die Berge auf die Richtung des Windes ausüben, ist von 2 weierlei Art: er ist ein mechanischer, der sich einfach darauf reducirt, dafs die von der Luft nicht wegschiebbaren Bergmassen den Strömungen besondere Wege anweisen; er ist aber auch ein dynamischer, insofern ein Berg oder ein Gebirge an und für sich Luftströmungen hervorruft und bei sonstiger Windstille eine Bewegung der Luft veranlafst, oder bereits vorhandenen Wind mehr oder weniger ändert. Unter diese letztere Klasse gehören vorzugsweise jene vou Bergen abhängigen Luftströmungen, die man in deın angränzenden Flachlande bemerkt, und sie sollen zunächst der Gegenstand der nachstehenden Untersuchung seyn. Selbstverständlich sind auch in dem vom Gebirge ab- 
bängigeu Flachlande die grofsen Strömungen der Luft vou Südwest nach Nordost und ungekehrt die hervorragenden, und die in Nachstehenden besprochenen sind nur in der Weise zu betrachten, wie man es etwa uit den Orvamenteu in Beziehung auf den ganzen Bau zu thun gewohnt ist. lu dem einem Gebirge nahen Festlande sind die Windverhältuisse in grofsen Ganzen von den anderweitigen uicht verschieden, aber daneben findet man noch mehr oder weniger beträchtliche Modificatiouen dieses grofsen Ganzen.

Wäre in Flachlande für sich beständig Windstille, so würde die Ermittelung des Gebirgseinflusses sehr ciufach seyn, denn es würde in diesem Falle ein Wind erregt, der entweder von der Beobachtungsstation zum Gebirge oder von diesem zur Station ginge. Sind jedoch schon andere Winde vorhanden, so werden diese nur modificirt. Erregt z. B. das Gebirge einen Südwind, so mufs nach dem Satze voin Parallelogramme der Kräfte die Gesammtbewegung der Luft eine unehr südliche werden; als sie aufserdem seyn würde. Soll die Rechnung strenge durchgeführt werden, so ist nothwendig, dafs die Geschwindigkeit der bewegten Luft bekannt sey; allein da diese Bedingung an keinem Orte der Erde erfüllt ist, so soll im Nachstehenden angenommen werden, dafs alle Winde gleich stark seyen, eine Aunabue, wegen welcher sich zu verantworten kaum nothwendig seyn wird, da sie gegenwärtig sämmtlichen Untersuchungen uber die Winde zu Grunde liegt.

Bekanntlich kann man nach dem von Lambert vorgeschlagenen Verfahren die Winde als Krăfte darstelleu, welche die Atmosphäre eines Ortes zu verrücken streben, und es läfst sich derjenige Wind bestimmen, der für sich allein dieselbe Wirkung ausüben wïrde, als alle beobachteten Winde zusammen, denn man braucht nur nach den Gesetzen von der Zusammenlegung der Krafte die Resultirende der einzeluen Seitenkräfte zu suchen. Dieses Verfahren benutzte ich insofern, als ich zwar die Componirenden aber nicht den mitlleren Wind bestimme. Hat z. B. ein Ort im Süden oder Norden ein Gebirge, dessen Einflufs zu be- 
stimmen ist, so suche ich die Projectionen auf die Südnordrichtung, da das Gebirge nur auf diese eine Aenderung her. vorbringt, während die Ostwes/projeclionen davon uur wenig oder gar nicht afficirt werden. Bezeichnet mav mit $A, B, C \ldots$ die Summe der beobachteten Nord-Nordost... winde, so ist:

$$
\begin{aligned}
& \left(\mathrm{S}-\mathrm{N}=E-A+(D+F-B-H) \cos 45^{\circ}\right. \text { und } \\
& (\mathrm{S}-\mathrm{W}-\mathrm{NO}) F-B+(G+E-A-C) \cos 45^{\circ} .
\end{aligned}
$$

Je gröfser die Zahl der beobachteten Winde ist, um so bedeutender wird die Summe der berechneten Projectionen seyn. Un ein einheitliches Maafs einzuführeu, gelten im Nachslehenden diejenigen Projectionen, welche neunzig Beobachtungen entsprechen, die sich also entziffern, wenn man ein Vierteljahr hindurch täglich Beobachtungen macht. Ist $n$ die Zahl sämmtlicher Windbeobachtungen, $p$ die arithmetische Summe der daraus berechneten Componirenden wach einer gegebenen Richtung, so werden die von mir angegebenen Ziffern dem Bruche $\frac{90 p}{n}$ entsprechen.

Als Beobachlungsstationen, die zur Ermittelung des Einflusses der Gebirge dienen sollen, sind solche Orte gewähl, welche in oder tiber dem Flachlande gelegen, auf der einen Seite nicht allzu weit von einem Gebirge entfernt sind. Die Gebirge und die Wasserflïchen sind ohne $Z$ weifel nicht die einzigen Localitäten, die einen Einflufs auf die Winde ausuben, und darum schränkt sich die Zahl der Beobachtungsstationen ziemlich ein, denn es dürfen nur möglichst wenig andere Umstănde vorhauden seyn. Ideal wäre eine Gebirgsmasse rings von einer grofsen überall gleichartigen Ebene umgeben, in welcher in gleicher Eutfernung voin Gebirge rings die Beobachtungsstationen zerstreut wären. Da aber dieses Ideal nirgends erfüllt ist, mufs man eben die Stationen nehmen wie sie sind. Mir sind nur die Punkte Bogenhausen bei München, Hohenpeifsenberg, Mailand, Karlsruhe und Mannheim bekannt, welche die geforderten Bedingungen möglichst erfüllen. Ist die Frage über die Gebirge erledigt, so mögen andere Localeinflüsse wie die der Waldunget, Haiden usw. an die Reihe kommen. 
A. Bogenhausen.

Die Beobachtungen über die Windverhältnisse von Bogenhausen sind von Lamont (Annalen für Meteorologie und Erdmagnetismus I - XII, Annalen der Köuigl. Sternwarte bei München I - XII, und monatliche und jährliche Pesultate der an der Königl. Sternwarte bei München von 1825 bis 1856 angestellten Beobachtungen) veröffentlicht. Man findet die Beobachtungen für alle geraden Stunden des Tages und der Nacht aus deu Jahren 1843 bis 1916 und für die geraden Stunden des Tages $\left(8^{\mathrm{b}} \mathrm{M}\right.$. bis $6^{\mathrm{b}} \mathrm{A}$.) aus den Jahren 1847 bis 1857. Bogenhausen liegt auf der bayerischen Hochebene fern von erheblichen Bergen und uördlich von der von Ost nach West ziehenden Alpenkettc. Der Rauuersparnifs wegen babe ich mich im Nachfolgenden stets auf die Untersuchung von Winter (December bis Februar), Sommer und das ganze Jahr beschräukt. Die Resultate der Beobachtungen sind folgende:

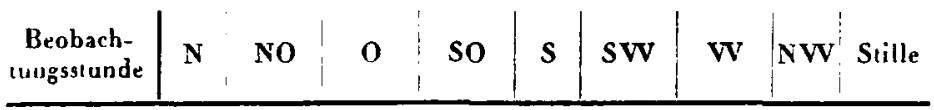

\begin{tabular}{|c|c|c|c|c|c|c|c|c|c|c|}
\hline \multicolumn{11}{|c|}{ Winter. } \\
\hline Morgens & $2^{b}$ & 6 & 24 & 49 & 16 & 1 & 56 & 78 & 1 & $6: 3$ \\
\hline & 4 & 6 & 19 & 40 & 9 & 8 & 62 & 87 & I & 67 \\
\hline & 6 & 2 & 26 & 41 & 17 & 11 & 87 & 110 & 7 & 47 \\
\hline & 8 & 22 & 102 & 176 & 126 & 84 & 356 & $3: 35$ & 55 & 98 \\
\hline & 10 & 21 & 116 & 206 & 119 & 63 & $30:$ & 355 & 66 & 99 \\
\hline & 12 & 25 & 171 & 221 & 82 & 37 & 249 & 369 & 75 & 93 \\
\hline Abends & 2 & 39 & 226 & 212 & 80 & 29 & 209 & 384 & 91 & 84 \\
\hline & 4 & 45 & 216 & 212 & 85 & 33 & 224 & 370 & 90 & 79 \\
\hline & 6 & 23 & 154 & 222 & 66 & 48 & 213 & 387 & 60 & 136 \\
\hline & 8 & 2 & 29 & 45 & 18 & 8 & 71 & 72 & - & 52 \\
\hline & 10 & 1 & 33 & 45 & 18 & 4 & 64 & 68 & 4 & 50 \\
\hline & 12 & 2 & 31 & 53 & 12 & 6 & 63 & 79 & - & 49 \\
\hline \multicolumn{11}{|c|}{ Sommer. } \\
\hline Norgens & $2^{h}$ & 4 & 13 & 31 & 16 & 7 & 122 & 106 & 4 & 60 \\
\hline & 4 & 3 & 17 & 14 & 13 & 8 & 196 & 52 & 10 & $\mathbf{5 5}$ \\
\hline & 6 & I & 23 & 16 & 17 & 5 & 243 & 27 & 11 & 22 \\
\hline & 8 & 33 & 85 & 182 & 88 & 36 & 411 & 377 & 100 & 68 \\
\hline & 10 & 50 & 125 & 222 & 69 & 17 & 287 & 400 & 161 & 49 \\
\hline & $1 \cdot 2$ & 86 & 170 & 197 & 46 & 13 & 225 & 404 & 208 & 31 \\
\hline \multirow[t]{6}{*}{ Abends } & 2 & 123 & 213 & 154 & 45 & 12 & 180 & 365 & 223 & 35 \\
\hline & 4 & 121 & 246 & 143 & 29 & 11 & 186 & 334 & 264 & 45 \\
\hline & 6 & 110 & 236 & 147 & 61 & 23 & 184 & 339 & 211 & 78 \\
\hline & 8 & 5 & 70 & 29 & 21 & 1 & 140 & 62 & 18 & \\
\hline & 10 & 6 & 33 & 29 & 23 & 8 & 126 & 81 & 4 & 55 \\
\hline & 12 & 6 & 16 & 34 & 12 & 10 & 131 & 97 & 5 & 65 \\
\hline
\end{tabular}




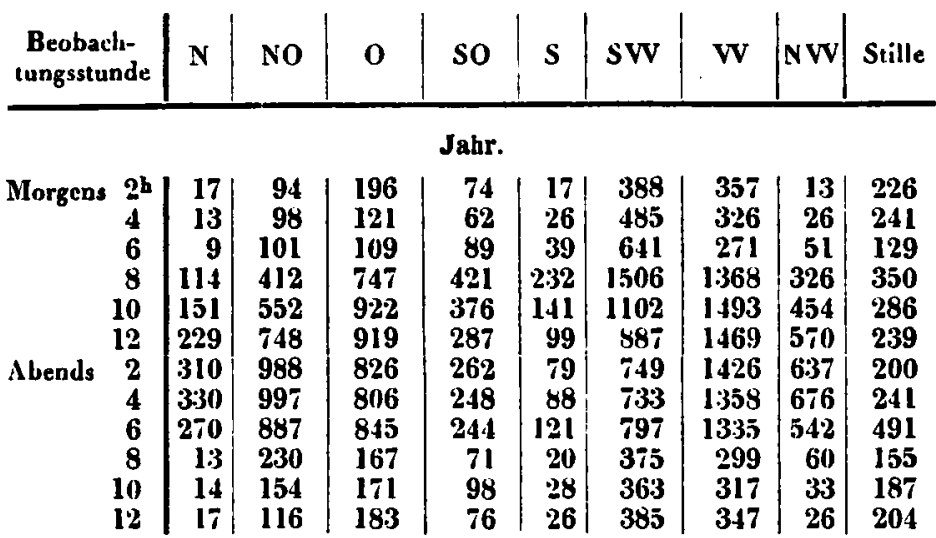

Berechnet man aus diesen Beobachtungen die Componirenden $(S-N)$ und $(W-O)$, so erhält man nachstehendes Resultat:

\begin{tabular}{|c|c|c|c|c|c|c|}
\hline \multirow{2}{*}{$\begin{array}{c}\text { Benbachtungs- } \\
\text { stunde }\end{array}$} & \multicolumn{2}{|c|}{ Vinter } & \multicolumn{2}{|c|}{ Sommer } & \multicolumn{2}{|c|}{ Jahr } \\
\hline & $(\mathrm{S}-\mathrm{N})$ & $(\mathrm{V}-\mathrm{O}-\mathrm{O}$ & $(S-N)$ & $(\mathrm{VV}-\mathrm{O})$ & $(S-N)$ & $(\mathrm{W}-0)$ \\
\hline $\begin{array}{rr}\text { Morgens } & 2^{\mathbf{b}} \\
4 \\
6 \\
6 \\
8 \\
10 \\
12 \\
12 \\
\text { Abends } \\
2 \\
4 \\
6 \\
8 \\
10 \\
12\end{array}$ & $\begin{array}{r}8,64 \\
11,46 \\
15,31 \\
19,40 \\
14,28 \\
4,91 \\
-1,98 \\
-0,66 \\
4,88 \\
14,67 \\
10,92 \\
10,71\end{array}$ & $\begin{array}{l}12,56 \\
21,60 \\
27,17 \\
19,17 \\
16,41 \\
13,49 \\
11,15 \\
11,11 \\
13,92 \\
13,32 \\
10,98 \\
1 \%, 25\end{array}$ & $\begin{array}{r}21,96 \\
32,70 \\
40,39 \\
14,68 \\
1,08 \\
-9,70 \\
-18,35 \\
-20,79 \\
-15,46 \\
11,68 \\
20,02 \\
22,02\end{array}$ & $\begin{array}{l}35,60 \\
39,73 \\
40,03 \\
28,30 \\
23,32 \\
23,51 \\
19,06 \\
20,54 \\
17,51 \\
19,71 \\
25,72 \\
34,27\end{array}$ & $\begin{array}{r}16,35 \\
20,09 \\
27,44 \\
15,76 \\
5,32 \\
-3,83 \\
-10,93 \\
-12,02 \\
-7,01 \\
7,60 \\
13,70 \\
15,30\end{array}$ & $\begin{array}{l}21,21 \\
29,18 \\
32,3.3 \\
21,82 \\
16,68 \\
14,02 \\
11,44 \\
10,97 \\
10,55 \\
14,68 \\
16,34 \\
20,80\end{array}$ \\
\hline
\end{tabular}

Man sieht aus den vorstehenden Ziffern, dafs die Sumunen der Projectionen der Winde einer von der Tagesperiode abbängigen Veränderung unterliegen, welche sich über die Meridian - und über die Ostwestrichtung erstreckt.

Was die Meridiaurichtung anbelangt, so ist die Zahl der südlichen Winde am Morgen ein Maximum, worauf sie sich zuerst rasch, dann langsamer bis Nachmittags vermindert; Nachmittags ist ein Minimum der Südwinde, dann nehmen dieselben zu und zwar an schnellsten am Abend, un bis 
zum Morgen den alten Stand einzunehmen '). Die Aeuderung der Projectionssummen beträgt, wenn man uur die Beobachtungsstunden berücksichtigt, und davon absieht, dafs die wahren Extreme daneben fallen künnen, im Winter 21,38 Einheiten, im Sommer 61,18, im Jabresdurchschnitte 39,46 , welch letzterer $W$ erth von dem arithmetischen Mittel der beiden ersteren nicht allzuweit abweicht. Die Grölsc der Aenderung ist also abhängig vou der Länge der Tage und wenn auch wegen mangelnder Beobachtungen über die Intensität der Winde das Gesetz, nach dem die Aenderungen vor sich geben, nicht festgestellt werden kann, so wird es wohl nicht unrichtig seyn, weun man anninmt, die Wirkung und mil ihr die Ursache der Aenderung sey im Sommer grölser als in Winter. Wenn die Aenderung in Winter 21,38, in Sominer 61,18 Einheiten beträgt, so glaube ich annehmen zu können, dafs die diese Aenderung bewirkende Ursache im Sommer mächtiger wirke als in Winter; es wäre aber zu weit gegangen, anzunehmen, dafs beidc Ursachen sich verhalten, wie 21,38 zu 61,18. Eine derartige Bestiumung liefse sich nur machen, wenn die jeweilige Geschwindigkeit der Strömungen bekannt wäre. Das Morgenmaximum der Südwinde ist im Winter um $8^{b}$, in Soinmer um $4^{\text {b }}$, und es ergiebt sich daraus eine sehr deutliche Analogie mit dem Gange der Tagestemperatur; doch treten bei dieser die beiden Extreme etwas früber auf.

Die vorstebende Erscheinung dürfte sich wohl sehr schwer oder gar nicht als eine allgemeine auf der Erde oder doch wenigstens auf unserer Halbkugel stattfindende nachweisen und erklăren lassen, und es muls daher die zu Bogenbausen beobachtete Aenderung der Winde als von Localverbaltuissen abhängig angenommen werden. Sucht man in der Gegend südlich und nördlich von München

1) Im Winter scheint boch ein zweites Minimum um $2^{\text {b }}$ Morgens zu seyn; da aber die andern Jahreszeiten nicht deutlich dafür sprechen, wie äberhaupt die Aenderungen in der Nacht nicht sehr deutlich sind, und da mir von den andern Stationen keine Nachtbeubachtungen bekannt sind, so soll diese 2 weite Periode für jetzt nicht weiter untersucht werden. 
nach dein Gegenstande, der die in der Meridianrichtung stattfindende Aenderung der Wiude hervorruft, so kann man diesen nur in dem südlich liegenden Gebirge finden, da der Norden nichts besonderes nachzuweisen hat. Vom Morgen an erhöht sich die Zahl der gegen das Gebirge ziehenden Winde, Abends nimmt sie wieder $a b$, und das Gebirge steht daher zu dem anliegenden Flachlande in derselben Beziebung, in der ,vir das Küstenland gegen das Meer finden. Das Gebirge entspricht der Kïste, das Flachland dem Meere und die vorstehende Aenderung des Windes ist dein Uebergange des Landwindes in Seewind und ungekehrt analog.

Denkt man sich um die Erde eine ideale Sphaeroïdfläche, deren Radius um ein Paar tausend Fufs gröfser ist, als der wirkliche der Erde, so geht diese durch einen Ocean von Luft, aus dem wie Inseln aus dem Mcere die Gebirge hervorragen. Auf diesen Inseln mufs bei der Insolation eine grölsere Wärmeentwicklung stattfinden, und die Folge davon werdert in den Gebirgen entspringende aufwärts gerichtete Strömungen seyn. Die Luft geht in die Höhe und wird von den Seiten wie von der Tiefe aus ersetzt. In der Nacht findet auf den Bergen durch stärkere Ausstrahlung eine bedeutendere Erkaltung der Luft und dann ein Herabströmen in die Ebene stalt.

Auch die nach der Ostwestrichtung berechneten Sunmen der Componirenden verändern sich im Laufe des Tages. Am Morgen, um die Zeit des Wärmeminimums sind die Westwinde am meisten vorwiegend, worauf sie immer uehr abnehnen, bis sie um die Zeit des Sonnenuntergangs ihr Minimum erreichen. Dann kehren sie bis zum Morgen zum alten Stande zurück. Auch hier ist die Veränderung in Sommer grölser als im Winter, aber weder in den einzelnen Jahreszeiten, noch im Jahresdurchschnitte ist sie so bedeutend wie die in der Meridianrichtung. Auch die Zeit des Einlretens schwankt weniger; letztere sind aber beide von deın Mittage weiter entfernt als die Extreme der Südnordrichtung. Da so die Wendepunkte sebr weit ausein- 
ander liegen (im Sommer 10 Stunden) und weil die Veranderung auch hier in den Morgenstunden am bedeutendsten ist, so bleibt für die Nachmittagsstunden nur wenig übrig.

Die Localverhältnisse der Ungebung von München bieten keinen Anhaltspunkt zur Erklärung dicser Erscheinung, da in Osten dasselbe Flachland ist, wie in Westen; dafür haben wir einen solchen an Hinmel in Laufe der Sonne. Aın Morgen liegen die wärmeren Gegenden östlich, am Nachunittag westlich von einem gegebenen Beobachtungsorte; da aber die Luft auf der Erdoberfliache stets dem wärmeren Orte zuströunt, so mufs auch die Zahl der in der Ostwestrichtung zichenden Winde mit dem Stande der Sonne sich ändern: die Ostwinde werden in Laufe des Tages zunehmen, und weil sich bis zum Morgen der ursprüngliche Stand wieder herstellen mufs, denn soust würden an Ende die Westwinde ganz ausbleiben, so werden in der Nacht die letzteren wieder zahlreicher. Da sich gegen diese Sälze von physikalischer Seite schwerlich viel einwenden läfst, so dürfte es zur Zeit wohl gestattet seyı, anzunehmen, dals die in München beobachtete Aenderung der Ostwestwinde wenigslens zum Theile auf den Lauf der Sonne zurückzuführen sey; aber anders würde sich die Sache gestalten, wenn man die Norm aufsuchen wollte, nach der die Aenderung vor sich geht, denn hier wirkt offenbar die Masse vieler in östlicher Richtung liegender Landstriche gegen die der westlichen; alle haben gleichzeitig verschiedene Tageszeiten also auch verschiedene Temperaturen, und wirken aus verschiedener Entfernung, so dafs es wohl schwer seyn dürfte, alle diese Verhältnisse auseinander zu suchen. Es ist übrigens auch das Gebirge hiebei thätig. Wäre südlich von München ein einziger Berg, so wăre nicht denkbar, wie dieser auf die Ostwestwinde einen Einflurs ausüben sollte; aber die Alpen sind nicht ein Berg, sondern eine von Ost nach West ziehende Kette. Am Vormittage wird der östlich von München ge legene Theil am meisten wirken, Nachmittags der westliche 
und es wird daher in der ersteren Tageszeit ein Nordwestwind in der zweiten ein Nordost vind hervorgerufen, deren Projectionen sich watürlich uuter die der übrigen Ostwestwinde mischen.

Da die Sonne Mittags südlicher steht als Morgens, so hönnte man versucht seyn, auch die Aenderung der Südnordwinde dew wechselnden Sonnenstande zuzuschrciben; allein bei näherer Betrachtung wird man finden, dafs die Annahme, es könue die beobachtete Aenderung der Südnordwinde auch ohne die Alpen stattfinden, unzulässig ist, denn in einem Flachlande ist die Temperaturdifferenz unter verschiedenen Breiten gelegener Orte lange nicht so bedeutend, als die solcher, deren luänge also deren Tageszeit verschieden ist; und da die Temperaturdifferenzen die Grundlage der Winde sind, könnte hier nur eine ganz unbedeutende Aenderung hervorgerufen werden. Die Beobachtung zeigt jedoch, dafs die Ostwestwinde lange nicht so bedeutend wechseln als die Südnordwinde.

\section{B. Hohenpei/senberg.}

Die Beobachtungen von Hohenpeifsenberg sind vou Lamont (Beobachtungen des meteorologischen Observatoriums auf dem Hohenpeifsenberge von 1792 bis 1850) veröffentlicht; sie beschränken sich auf die Stunden $7^{\text {h }}$ Morgens, $2^{\text {h }}$ und $9^{\text {h }}$ Abends. Die Station Hohenpeifsenberg liegt auf einem 3016 Fufs hohen isolirten Gipfel, der auf denselben Flachlande aufsitzt wie München; er ist etwas näher an Gebirge, aber sonst unter denselben Verbältnissen. Nachstehend folgen die Beobachtungen der zehn Jahrgänge $1841-1850$.

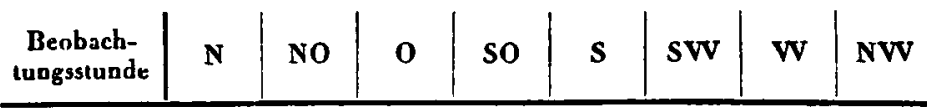

Winter.

\begin{tabular}{l|l|l|l|l|l|l|l|l}
$\mathbf{7 h}$ & $\mathbf{1 5}$ & $\mathbf{1 3 6}$ & $\mathbf{4 1}$ & $\mathbf{9 7}$ & $\mathbf{5 8}$ & $\mathbf{2 5 9}$ & $\mathbf{2 3 4}$ & $\mathbf{6 2}$ \\
$\mathbf{2}$ & $\mathbf{3 7}$ & $\mathbf{1 6 6}$ & $\mathbf{5 2}$ & $\mathbf{7 1}$ & $\mathbf{6 5}$ & $\mathbf{2 4 1}$ & $\mathbf{2 1 7}$ & $\mathbf{6 3}$ \\
$\mathbf{9}$ & $\mathbf{3 7}$ & $\mathbf{1 3 2}$ & $\mathbf{5 5}$ & $\mathbf{7 2}$ & $\mathbf{5 5}$ & $\mathbf{2 3 6}$ & $\mathbf{2 6 2}$ & $\mathbf{5 3}$
\end{tabular}




\begin{tabular}{r|l|l|l|l|l|l|l|l}
$\begin{array}{c}\text { Beobach- } \\
\text { tungsstunde }\end{array}$ & $\mathrm{N}$ & No & $\mathrm{O}$ & so & $\mathrm{s}$ & $\mathrm{SWV}$ & $\mathrm{VV}$ & $\mathrm{NWV}$ \\
\hline
\end{tabular}

Sommer.

\begin{tabular}{l|l|r|r|r|r|r|r|r} 
7h & 23 & 90 & 69 & 92 & 60 & 231 & 252 & 101 \\
$\mathbf{2}$ & 78 & 225 & 57 & 24 & 25 & 146 & 234 & 131 \\
9 & 53 & 172 & 58 & 57 & 53 & 171 & 272 & 84
\end{tabular}

Jahr.

\begin{tabular}{l|r|l|l|l|l|l|l|l}
$\mathbf{7}^{\mathrm{b}}$ & $\mathbf{7 7}$ & $\mathbf{4 7 1}$ & $\mathbf{2 4 5}$ & $\mathbf{4 3 3}$ & $\mathbf{2 1 3}$ & $\mathbf{9 5 3}$ & $\mathbf{8 9 9}$ & $\mathbf{3 2 7}$ \\
$\mathbf{2}$ & $\mathbf{2 1 2}$ & $\mathbf{8 7 3}$ & $\mathbf{2 1 6}$ & $\mathbf{2 1 2}$ & $\mathbf{1 6 6}$ & $\mathbf{7 0 2}$ & $\mathbf{8 6 5}$ & $\mathbf{4 0 5}$ \\
$\mathbf{9}$ & 167 & $\mathbf{7 0 8}$ & $\mathbf{2 1 6}$ & $\mathbf{2 6 1}$ & $\mathbf{2 2 0}$ & $\mathbf{7 6 8}$ & $\mathbf{1 0 1 8}$ & $\mathbf{2 9 : 3}$
\end{tabular}

Die aus diesen Beobachtungen abgeleiteten Projectionen sind:

\begin{tabular}{|c|c|c|c|c|c|c|}
\hline \multirow{2}{*}{$\begin{array}{l}\text { Beobach. } \\
\text { tungs- } \\
\text { stunde }\end{array}$} & \multicolumn{2}{|c|}{ VVinter } & \multicolumn{2}{|c|}{ Sommer } & \multicolumn{2}{|c|}{$\mathrm{J}_{\mathrm{ahhr}}$} \\
\hline & $(S-N)$ & $(\mathrm{W}-\mathrm{O})$ & $(S-N)$ & $(W-0)$ & $(S-0)$ & $(V \mathrm{~V}-(0)$ \\
\hline $\begin{array}{l}7^{\mathrm{h}} \\
2 \\
9\end{array}$ & $\begin{array}{r}15,44 \\
7,65 \\
10,47\end{array}$ & $\begin{array}{l}25,47 \\
21,19 \\
26,25\end{array}$ & $\begin{array}{r}12,78 \\
-18,05 \\
-\quad 1,94\end{array}$ & $\begin{array}{l}28,34 \\
19,25 \\
22,73\end{array}$ & $\begin{array}{r}14,35 \\
-\quad 7,48 \\
1,80\end{array}$ & $\begin{array}{l}22,69 \\
16,38 \\
21,37\end{array}$ \\
\hline
\end{tabular}

Die Vergleichung dieser Ziffern mit den Resultaten der Münchener Beobachtungen ergiebt, dafs der Charakter der Aenderung der Winde an beiden Orten derselbe sey; am Tage nehmen die Nordwinde wie die Ostwinde zu. Hohenpeifsenberg liegt wie München nördlich von den Alpen und dort geht wie hier die Sonne im Osten auf. Trotz dieser allgemeinen Aebulichkeit, ist das Verhalten des Win. des nicht an beiden Orten das nämliche. Die Verschiedenheiten beider Orte in Bezug auf die Südnordwinde sind vorzugsweise folgende:

1) Die absolute Gröfse der Aenderung der Winde ist in München bedeutender als in Hohenpeifsenberg.

2) Die Aenderung von $7^{\text {b }}$ bis $2^{\text {h }}$ ist, im Vergleiche zu Bogenhausen, auf dem Peifsenberge verhältnifsmäfsig grölser als von $2^{\text {h }}$ bis $9^{\text {h }}$.

Der Peifsenberg ist selbat ein Berg und wird wenn auch seiner geringeren Masse entsprechend in geringerem Grade als die Alpen doch mit einiger Stärke seinen Einflufs auf 
die Winde der Gegend ausüben, und das Flachland zwischen Hohenpeifsenberg und den Alpen wird sich in den Einflufs beider theilen. Die Südseite des Peifsenberges sucht die Luft zu einer Bewegung zu veranlassen, welche an dem Abhange in die Hüle geht, also eine Richtung von Süd nach Nord hat, während dic Nordseite des Berges den entgegengesetzten Einflufs hat. Die Südscite wird mehr erwärmt, ilsr Wind wird daber verschlagen und es resultirt daher, dafs von der Nordseite wie von der Südseite Winde an dem Gipfel zusammentreffen, von deneu die südlichen stärker sind. Olne den Berg würden also auf der Station Mitlags weniger Südwinde seyn und die Grölse der Projectionen würde sich daber ändern, wenn man die Bergwirkung abziehen könnte. Setzen wir diese zu sechs Einheiten, so würde die Projectionssunme in Jahresdurchschnitte $-13,48$ betragen, und die Aenderung ron $7^{\mathbf{h}}-2^{\mathbf{b}}$ würde 27,83 , die von $2^{\text {b }}-9^{\text {h }}$ würde 14,28 belragen. Die absolute Gröfse der Aenderung würde mithin bedeutender und die Nachnitlagsänderung würde verhältnifsmälsig mehr wachsen als die des Vormittags. Beide würden sich verhalten wie 14,28 zu 27,83 , während sie so 9,28 zu 21,83 sind. Es kann auch aufserdem die durch die Alpen hervorgerufene Strömung in der Hühe kleiner seyn als in der Tiefe. Die auf den Bergen aufsteigende Luft mufs in der Höhe über der südbayerischen Ebene einen Strom von Süd nach Nord hervorrufen, während unten ein Wind von Nord

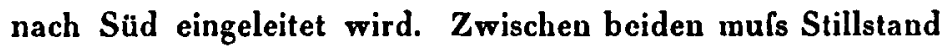
seyn, und wemn man von unten aufsteigend sich diesem Nullpunkte năbert, so mufs die Bewegung der Luft allmählig abnebmen. Bringt man in die balbgeöffnete Thüre eines geheizten Zinmers eine Kerzenflamme, so zeigt die letztere am Boden eine in das Zimmer eindringende Strömung, die abnimmt, wenn man mit der Kerze höher rückt, etwa in der Mitte steht die Flamme gerade und oben zeigt sie auswärts. Dieser Fall bietet ein Analogon zu den Verhältnissen, die man bei den Gebirgen beobachtet, und auf dem Peifsenberge würde man daher auch ohne die Wirkung des 
Berges selbst eine geringere Aenderung der Windrichtung wahrnehmen, als diese in München beobachtet wird.

Auch in Beziehung auf die Ostwestrichtung findet man die Verschiedenheiten:

1) Die absolute Gröfse der Aenderung ist in Hohenpeifsenberg kleiner als in München.

2) Die Vornittagswirkung ist wenigstens in Jahresdurchschuilte verbältuifsmäilsig grölser als die Aenderung des Nachmittags.

Die Aenderung der Ostwestwinde rührt von den Wärmcunterschieden her, welche an Orten verschiedener Länge durch die Verschiedenheit der Tageszeit bedingt wird. In der Höbe wechselt die Temperatur in Laufe des Tages weniger als in der Tiefe'), mebrere neben einander liegende Punkte von ungleicher Länge anterscheiden sich hinsichtlich der Wärme in der Höhe weniger als unten, und darum muls auch die von der geringeren Wärmedifferenz abhäıgige Luftströmung oben kleiner seyı.

Bestrablt die Sonne an Vormittage den Berg, so wird sie auf der Morgenseite einen Ostwind, auf der Abendseite einen Westwind erregen, die auf dem Gipfel sich treffen, von denen aber der Ostwind der stärkere ist. Auch die Sonne hilft vom Morgen an zu den Ostwinden, sie wird also von dem Berge unterstützt, und ohne diesen würde die Aenderung vou $7^{b}-2^{b}$ noch kleiner. Bis zum $A$ bend stelit sich, da die Sonne Nachnittags die Westwinde begünstigt, das ursprüngliche Verbältnils wieder her. Würden sich (also nach Abzug der Bergwirkung) die Projectionen von $7^{\mathrm{b}}-2^{\mathrm{b}}$ nur von 22,69 auf 19,38 statt auf 16,38 ändern, so wäre die ganze Aenderung nur 3,31 Einheiten, und wenn sich bis $9^{\text {h }}$ das ursprüngliche von 21,37 wieder herstellen würde, da mittlerweile die Bergwirkung aufgehört hat, so würde sich die Aenderung $2^{\text {b }}-9^{\text {h }}$ auf 1,99 Einheiten belaufen, und die Vormittagsänderung wäre im Verbältnifs zu der Nachınittagsänderung grölser, wie dieses

1) Dove in den Tabellen und amtlichen Nachrichten über den preufsischen Staat V. 
auch in München stattindet. Ich mufs übrigens darauf aufmerksam machen, dafs mit dieser Erklärung der Thatsache noch nicht alle Einwürfe gehoben sind, denn der Sommer bietet die umgekehrte Erscheinung, weil dort die Nachnittagsänderung verhältnifsmäfsig kleiner ist, und es müssen daher noch weitere Einwirkungen stattfinden, die ich jedoch zur Zeit nicht kenne.

\section{Mailand.}

Von Mailänder Windbeobachtungen sind mir nur drei Jahrgänge bekannt: zwei davon ( 1842 und 1813) unit den Beobachtungsstunden $8^{\mathrm{h}}$ Morgens, $2^{\mathrm{h}}$, $8^{\mathrm{h}}$ und $11^{\mathrm{h}}$ A bends finden sich in Lamont's Annalen für Meteorologie und Erdwagnetismus VII und XI, einen dritten Jahrgang (1844) hat mir Hr. Lam ont aus einem Briefe des Hrn. Capelli, Adjuncten an der Mailänder Sternwarte, mitgetheilt. Hier sind die Beobachtungsstunden $6^{\text {h }}$ und $9^{\text {b }}$ Morgens und $3^{\text {h }}$ und $9^{\text {b }}$ Abends. Es wurden in Mailand auch die Zwischenwinde NNO, ONO usw. angegeben. Der Gleichförmigkeit wegen sind diese Winde je zur Hälfte den Nachbarrichtungen zugezählt, wie dieses ziemlich allgemein üblich ist. (Vergl. Kä m t z Meteorologie I, 153). Die Mailäuder Beobachtungen umfassen so acht Beobachtungsstunden, die aber aus verschiedenen Jahrgängen stammen, weshalb sich auch in der Projectionstabelle einige Kreuzsprünge befinden, die wohl ausgefallen wären, wenn ich eine gröfsere Reihe von Jahren hätte benutzen köunen. Mailand liegt bekanntlich in der lombardischen Ebene im Süden der Alpen, und letztere müssen daher eine Einwirkung ausüben, welche der in München beobacbteten entgegengesetzt ist

Die Beobachtungen sind folgende: 


\begin{tabular}{c|c|c|c|c|c|c|c|c}
$\begin{array}{c}\text { Beobacl- } \\
\text { tungsstunde }\end{array}$ & N & No & o & so & s & sw & W & NW \\
\hline
\end{tabular}

Winter.

\begin{tabular}{|c|c|c|c|c|c|c|c|c|c|}
\hline Morgens & $6^{b}$ & 12 & 15 & 15 & 4,5 & 4,5 & 4,5 & 18 & 17,5 \\
\hline & 8 & 30,5 & 29,5 & 22,5 & 6 & 4 & 19 & & \\
\hline & 9 & 10,5 & 17 & 19,5 & 6 & - & 7,5 & 13 & 6.5 \\
\hline bends & 2 & 17,5 & 17 & 14 & 21 & 13,5 & 46 & 23 & \\
\hline & 3 & 11 & 6,5 & 10 & $\mathbf{5 , 5}$ & 4 & & & \\
\hline & 8 & 26 & 30 & 16 & 7 & 5 & 27,5 & & \\
\hline & 9 & 14 & 12,5 & & 2 & 4 & 7 & 16,5 & 20,5 \\
\hline & 11 & 27 & $34, \mathbf{3}$ & 26,5 & 8 & 3 & 16 & 28,5 & \\
\hline
\end{tabular}

Sommer.

\begin{tabular}{|c|c|c|c|c|c|c|c|c|c|}
\hline Morgens & $6^{b}$ & 15 & 21 & 16 & 2,5 & $\mathbf{4 , 5}$ & 6,5 & 10,5 & 16 \\
\hline & 8 & 11 & 52,5 & 22 & 23 & 8 & 29 & 9,5 & $24, \overline{5}$ \\
\hline & 9 & 4 & 13 & $24, \bar{j}$ & 11,5 & 7,5 & 15 & 6,5 & 8 \\
\hline & 2 & 11 & 21 & 27 & 28 & 16,5 & 34 & 16 & 19,5 \\
\hline & 3 & 4 & $\mathbf{5}$ & 19 & 3 & & 19,5 & 19 & 8,5 \\
\hline & 8 & 19,5 & 27,5 & 15 & 29,5 & 18 & & 17 & 18,5 \\
\hline & 9 & 10 & $i$ & 16 & 4 & $\mathbf{5}$ & 18,5 & 15 & 1.4 \\
\hline & 11 & 25,5 & 40 & 11,5 & 12 & 7 & 24 & 24 & 32 \\
\hline
\end{tabular}

Jahr.

\begin{tabular}{|c|c|c|c|c|c|c|c|c|c|}
\hline Morgens & $6^{\mathrm{h}}$ & $5,3,5$ & 79 & 74 & 17 & 13 & 19,5 & 50,5 & 59,5 \\
\hline & 8 & 84 & 165 & 94,5 & 6.5, 5 & 39 & & 66,5 & 13.5 \\
\hline & 9 & 29 & 61 & 99 & 31 & 14 & 41 & 42 & 45 \\
\hline A bends & 2 & $\mathbf{5 5 , 5}$ & 70 & & 91,5 & & 154 & & 95,5 \\
\hline & 3 & 21 & 26 & & 35 & 33 & & & 41 \\
\hline & 8 & $\mathbf{7 3 , 5}$ & 105 & 80 & 80,5 & 48,5 & 106 & 94 & 101 \\
\hline & 9 & 49 & $49, \mathbf{3}$ & & 14 & & & & 68 \\
\hline & & 111, & 171 & & 42 & 19,5 & 70 & & 135 \\
\hline
\end{tabular}

Hieraus ergeben sich die Projectionssummen:

\begin{tabular}{|c|c|c|c|c|c|c|}
\hline \multirow{2}{*}{$\begin{array}{c}\text { Beobachtungs- } \\
\text { stunde }\end{array}$} & \multicolumn{2}{|c|}{ VVinter } & \multicolumn{2}{|c|}{ Sommer } & \multicolumn{2}{|c|}{ Jahr } \\
\hline & $(S-N)$ & $(\mathrm{w}-\mathrm{O})$ & $(S-N)$ & $(\mathrm{VV}-\mathrm{O})$ & $(S-N)$ & $(W-0)$ \\
\hline $\begin{aligned} & \text { Morgens } 6^{\mathbf{h}} \\
& \mathbf{8} \\
& \mathbf{9} \\
& \text { Abends } \mathbf{2} \\
& \mathbf{3} \\
& \mathbf{8} \\
& \mathbf{9} \\
& \mathbf{1 1}\end{aligned}$ & $\begin{array}{r}-23,59 \\
-28,45 \\
-24,64 \\
+\quad 6,74 \\
-8,24 \\
-20,55 \\
-27,22 \\
-28,02\end{array}$ & $\begin{array}{r}4,66 \\
11,53 \\
-5,79 \\
+16,28 \\
-22,50 \\
14,85 \\
14,67 \\
3,53\end{array}$ & $\begin{array}{r}-29,64 \\
-10,09 \\
+\quad 7,39 \\
10,77 \\
13,51 \\
2,39 \\
-4,44 \\
-22,48\end{array}$ & $\begin{array}{r}6,07 \\
-14,03 \\
-19,06 \\
-\quad 4,07 \\
+13,79 \\
-3,99 \\
+14,20 \\
7,84\end{array}$ & $\begin{array}{r}-27,70 \\
-19,75 \\
-\quad 9,59 \\
+9,12 \\
+10,30 \\
-\quad 5,01 \\
-19,57 \\
-29,09\end{array}$ & $\begin{array}{r}-8,73 \\
-5,42 \\
-14,97 \\
+9,01 \\
+10,93 \\
+3,85 \\
+8,21 \\
-0,84\end{array}$ \\
\hline
\end{tabular}

Betrachtet man zuerst die Aenderungen der Winde, die in der Meridianrichtung vor sich gehen, so zeigt sich alsbald, dafs in Mailand der entgegengesetzte Fall von dem

Poggendorf's Annal. Bd. CXVI. 
in München beobachteten stattfindet. In München nehmen rom Morgen an die Nordwinde, in Mailande die Südwinde zu. Auch in Mailand treten die Extreme im Winter näher gegen Mittag als im Sommer. Die Gröfse der Schwankungen ist in Mailand in Winter gröfser, in Somner kleiner als in München, was den dortigen geringeren Schwankungen der Tageslänge entspricht. Stellt man die Resultate der Meridianrichtung beider Orte aus dem Jahresdurchschnitte zusammen, nachdem man für München dic Werthe der ungeraden Stunden so gut als möglich interpolirt hat, so ergiebt sich:

$\begin{array}{rrrr} & & \text { München. } & \text { Mailand. } \\ \text { Morgens } & 6^{\text {h }} & +27,44 & -27,70 \\ 8 & +15,76 & -19,75 \\ \text { Abends } & 9 & +10,38 & -9,59 \\ 2 & -10,39 & +9,12 \\ 3 & -12,23 & +10,30 \\ 8 & +7,60 & -5,01 \\ 9 & +11,46 & -19,57 \\ 11 & +14,82 & -29,09\end{array}$

Man siebt hieraus, dafs mit Ausuahine von $9^{\mathrm{h}}$ und $11^{\mathrm{b}}$ Abends die absoluten Grölsen wenig verschieden, die Zeichen aber die entgegengesetzten sind. Es ist übrigens nicht zu übersehen, dafs die Anzahl der Beobachtungsjahre in Mailand nicht grofs ist; wäre dieselbe bedeutender, so würden sich aller Wahrscheinlichkeit nach Aenderungen in den Ziffern ergeben, doch ist nicht wohl anzunehmen, dafs der so deutlich ausgesprochene Typus der Aenderungen eine wesentliche Veränderung erleiden werde.

Während in Beziehung auf die Meridianrichtung nicht wohl ein Zweifel seyn kann, dafs die Schwankungen durch die Alpen hervorgebracht werden, zeigt die Ostwestrichtung in den einzelnen Jahreszeiten nur sehr unregelmäfsige Aenderungen des Windes, die auch bei dem Jahresdurchschnitte nicht verschwinden, und es läfst sich nur sehen, dafs in Mailand vom Morgen bis Abend nicht wie in München eine Znnahme, sonderin eine Abnahme der Ostwinde statt- 
findet. Wenn auch aus dem oben angegebenen Gruude einige Unregelmälsigkeiten in dem Gange der Projectionen erwartet werden konnten, so sind diese doch auf der Ostwestrichtung in Vergleiche mit der Meridianrichtung so bedeutend, dafs man eine andere Wirkung vermuthen kann, welche die des wechselnden Standes der Sonne verdeckt. Sucht man nun auf einer guteu Landkarte die Lage Mailands gegen die Alpen festzustellen, so ergiebt sich alsbald, dafs die Wirkung des Gebirges in Mailand nicht wohl in der Meridianrichtung vor sich gehen kann, denn im Nordosten der Stadt dringen dic Berge bis Bergamo nach Süden vor, während in Nord und Nordwest nicht nur das Gebirge zurückweicht, sondern auch an deren Stelle als Lago di Como, Lago di Lugano und Lago maggiore Wasserflächen treten, welche die nach jenen Richtungen slattfindende Bergwirkung wenigstens theilweise aufheben. Das Gebirge wird also so wirken, als sey es in einem Punhte concentrirt, der (von Mailand aus) zwischen $\mathbf{N}$ und $\mathbf{O}$ gelegen ist. Projicirt man die Winde auf die Meridian - und die Ostwestrichtung, so wird erstere die Südnordcomponirende der Bergwirkung geben, während letztere die Ostwestcomponirende der Berg - und die ganze Sonnenwirkung enthält. In dem Meridiane ist sohin nur eine Wirhung thätig, weshalb dort auch die Schwankungen ziemlich regelmäfsig sind; senkrecht auf dem Meridian dagegen sind zwei Kräfte thätig, die einauder entgegengesetzt wirken und ihre Wendepunkte zu verschiedenen Zciten haben. Daraus lärst sich wenigstens zum Theile das Durcheinander in der Ostwestcolumne erklären, das übrigens auch abnehmen würde, wenn die Zahl der Beobachtungsjahre eine gröfsere wäre, wie man durch Vergleichung des Jabresdurchschnittes unit den einzelnen Jahreszeiteu leicht erkennen kann. Bedenkt man, dafs durch die Sonne ein Theil der Alpenwirkung fiir Mailand aufgehoben wird, und dafs der letzteren Südnordcomponirende schon so grofs ist, als die ganze Wirkung in München, so ergiebt sich, dals dieselbe südlich der Alpen gröfser seyn müsse als in gleicher Entfer- 
nung nördlich (die Entfernung beider Städte von den Bergen ist nicht sehr verschieden) was sich daraus erklärt, dafs die Alpen weiter über das südliche Tiefland berausragen, als über die nördliche Hochebene.

D. Karlsmihe.

Die Beobachtungen über die Windverbăltnisse von Karlsruhe, welche mir zu Gebole standen, sind die Jahrgänge 1841, 1812 und 1813 und sind den La no $n$ t'schen Anua. len für Meteorologie und Erdmagnetismus IV, VII und XI entuommen. Die Beobachtungsstunden sind $7^{\text {b }}, 2^{\text {b }}$ und $9^{\text {h }}$. Karlsruhe liegt in der Rheinebene und hat das Gebirge nicht in der Meridianrichtung wie die drei vorhergehenden Stationen, sondern in Osten. Die beobachteten Winde sind :

\begin{tabular}{|c|c|c|c|c|c|c|c|c|}
\hline $\begin{array}{l}\text { Beobach- } \\
\text { tungsstunde }\end{array}$ & $\mathbf{N}$ & No & 0 & so & $\mathbf{S}$ & SW & $\mathbf{W}$ & $\mathbf{N V}$ \\
\hline \multicolumn{9}{|c|}{ Winter. } \\
\hline $\begin{array}{l}\mathbf{7 b}^{\mathbf{h}} \\
\mathbf{2} \\
\mathbf{9}\end{array}$ & $\begin{array}{l}24 \\
20 \\
15\end{array}$ & $\begin{array}{l}62 \\
58 \\
87\end{array}$ & $\begin{array}{r}17 \\
18 \\
7\end{array}$ & $\begin{array}{r}11 \\
1 \\
1\end{array}$ & $\begin{array}{r}20 \\
12 \\
8\end{array}$ & $\begin{array}{l}117 \\
119 \\
127\end{array}$ & $\begin{array}{l}12 \\
26 \\
15\end{array}$ & $\begin{array}{r}7 \\
10 \\
10\end{array}$ \\
\hline \multicolumn{9}{|c|}{ Sommer. } \\
\hline $\begin{array}{l}7^{b} \\
2 \\
9\end{array}$ & $\begin{array}{l}17 \\
17 \\
17\end{array}$ & $\begin{array}{l}60 \\
39 \\
68\end{array}$ & $\begin{array}{r}23 \\
16 \\
9\end{array}$ & $\begin{array}{r}16 \\
6 \\
6\end{array}$ & $\begin{array}{l}20 \\
17 \\
14\end{array}$ & $\begin{array}{l}119 \\
112 \\
125\end{array}$ & $\begin{array}{l}11 \\
42 \\
21\end{array}$ & $\begin{array}{l}10 \\
27 \\
16\end{array}$ \\
\hline \multicolumn{9}{|c|}{ Jahr. } \\
\hline $\begin{array}{l}7^{h} \\
2 \\
9\end{array}$ & $\begin{array}{l}80 \\
83 \\
72\end{array}$ & $\begin{array}{l}290 \\
228 \\
3\lfloor 3\end{array}$ & $\begin{array}{l}75 \\
72 \\
29\end{array}$ & $\begin{array}{l}47 \\
26 \\
20\end{array}$ & $\begin{array}{l}79 \\
52 \\
40\end{array}$ & $\begin{array}{l}447 \\
431 \\
505\end{array}$ & $\begin{array}{r}48 \\
128 \\
59\end{array}$ & $\begin{array}{l}29 \\
75 \\
57\end{array}$ \\
\hline
\end{tabular}

Berechnet unan hieraus die Projectionen nicht nur nach den zwei Hauptrichtungen, sondern auch nach den (SW-NO) und (NW-SO) entsprechenden, so ergiebt sich:"

\begin{tabular}{c|c|c|c|c}
$\begin{array}{c}\text { Beobachtungs- } \\
\text { stunde }\end{array}$ & $(S-N)$ & $(S V W-N O)$ & $(W-O)$ & $(N V-S O)$ \\
\hline & Winter. \\
ib & 12,57 & 16,21 & 10,35 & $-1,57$ \\
2 & 11,00 & 20,33 & 17,75 & 4,77 \\
9 & 4,97 & 13,57 & 14,22 & 6,51
\end{tabular}




\begin{tabular}{|c|c|c|c|c|}
\hline $\begin{array}{l}\text { Beobachtuugs- } \\
\text { slunde }\end{array}$ & $(S-N)$ & $(\mathrm{SVV}-\mathrm{NO})$ & $\left(\mathrm{V} \mathrm{V}_{-} \mathrm{O}\right)$ & $(\mathrm{NWV}-\mathrm{SO})$ \\
\hline \multicolumn{5}{|c|}{ Sommer. } \\
\hline $\begin{array}{l}i^{b} \\
2 \\
9\end{array}$ & $\begin{array}{r}15,96 \\
11,99 \\
9,86\end{array}$ & $\begin{array}{r}17,16 \\
29,79 \\
20,66\end{array}$ & $\begin{array}{r}8,31 \\
30,15 \\
19,36\end{array}$ & $\begin{array}{r}-5,41 \\
12,84 \\
6,72\end{array}$ \\
\hline \multicolumn{5}{|c|}{ Jahr. } \\
\hline $\begin{array}{l}7^{b} \\
2 \\
9\end{array}$ & $\begin{array}{r}10,09 \\
6,40 \\
6,38\end{array}$ & $\begin{array}{l}11,28 \\
18,14 \\
15,66\end{array}$ & $\begin{array}{r}5,86 \\
19,25 \\
15,78\end{array}$ & $\begin{array}{r}-2,99 \\
9,08 \\
6,65\end{array}$ \\
\hline
\end{tabular}

Diese Zablen zeigen die gröfsten Aenderungen in deu Richtungen ( $\mathrm{W}-\mathrm{O}$ ) und $\mathrm{SW}-\mathrm{NO}$ ). Die westlichen Winde sind am Morgen an wenigsten zahlreich und nehmen bis $2^{\text {b }}$ zu bis $9^{\text {h }}$ wieder ab. Im Sommer ist die Aenderung gröfser als im Winter. Die Veränderung im Stande der Sonne sollte die Wirkung baben, dafs vom Morgen an die Westwinde zunehmen; aber die östlich liegenden Berge heben die Somnenwirkung nicht nur auf, sondern richten aufserdem noch den Wind in ihrem Sinne. Dic Aenderung nach (NW-SO) ist gröfser als die nach (SW-NO); es wirkt also das Gebirge nicht rein ostlich sondern etwas südlich. Die Massenentwicklung des Schwarzwaldes ist auch in der That sudüstlich von Karlsruhe gröfser als nordöstlich.

E. Mannheim.

Kä $\mathrm{mtz}$ giebt in ıseiner Meteorologie I. 217. als das Resultạt zwölfjähriger Beobachtungen von Mannheim folgende mittlere Windrichtungen an:

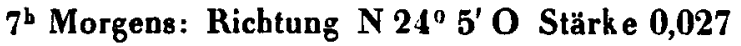

$2^{\text {h }}$ Abends: $\quad-\quad S 74^{\circ} 32^{\prime} W-0,134$

$9^{\mathrm{b}}$ Abends: $\quad-\quad \mathrm{S} 36^{\circ} 4^{\prime} \mathrm{W} \quad-0,010$

Werden diese mittleren Werthe in die Componirenden zerlegt, so ergiebt sich bei der Reduction auf 90 Tage: 


\begin{tabular}{|c|c|c|c|c|}
\hline $\begin{array}{l}\text { Beobachilungs- } \\
\text { stunde }\end{array}$ & $(S-N)$ & $(S W V-N O)$ & $(v v-0)$ & $(\mathrm{NW}-\mathrm{SO})$ \\
\hline $\begin{array}{l}7^{h} \\
2 \\
9\end{array}$ & $\begin{array}{r}-2,22 \\
3,22 \\
0,73\end{array}$ & $\begin{array}{r}-2,27 \\
10,49 \\
0,49\end{array}$ & $\begin{array}{r}-0,99 \\
11,62 \\
0,53\end{array}$ & $\begin{array}{r}+0,87 \\
5,95 \\
-0,14\end{array}$ \\
\hline
\end{tabular}

Hier sind die gröfsten Aenderungen nach den Richtungen (W-O) und (SW-NO), und zwar wehmen die Westwinde zu, zeigen also ein Gebirge im Osten an; es ist der Wechsel nach (SW-NO) grölser als nach (NW-SO); also tritt hier der entgegengesetzte Fall von dem in Karls rube beobachteten ein, und das Gebirge ist daher auch nicht rein östlich, sondern etwas gegen Norden (der Odenwald). Zwischen Karlsruhe und Mannbeim ist die Einsenkuug in dern östlichen Höhenzuge, durch den die Eisenbahn von Stuttgart nach Bruchsal geführt wurde.

Die absulute Grörse der Projectionen.

Wenn es nach dem Vorstehenden wobl als sicher anzunehmen ist, dals die Gebirge einen deutlichen Einflufs auf die Winde ausüben, so lä[st sich daraus noch nicht cntscheiden, ob diese Wirkung blofs die Folge bei Nacht herabkommender, ob blofs bei Tage aufsteigender Luftströıne sey. Es kann das Phänomen ron einer einzigen der obengenannten Ursachen berrühren, es können aber auch beide thätig seyn, und es kann hier ein ähnlicher

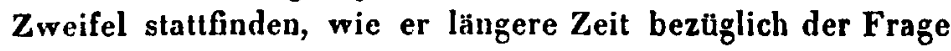
herrschte, ob es zwei sich entgegengesetzte Elecktricitäten oder nur eine gebe. Zur Beantwortung der vorstehenden Frage darf man sich nicht auf die Untersuchung der Aenderung der Projectionen beschränken: es muls auch deren absolute Grö/se berücksichligt werden.

Wären die Alpen nicht vorhanden, so würde an den Stationen München und Mailand irgend ein Wind herrschen, der eine Projection auf die Meridiaurichtung geben würde. Diese Projection wäre in Laufe des Tages wenig veränderlich, und sie wäre auch an beiden Orten ziemlich 
gleich grofs, denn die Entfernung derselben von ejnander ist nicht allzu bedeutend, und auf dem hohen Meere geben die Breiten $45^{\circ}$ und $48^{\circ}$ keine grofsen Differenzen. Hätten die Alpen keinen eigentlichen Nachtwind, also nur den durch Aufsteigen der Luft hervorgerufenen Tagwind, so mülste am Morgen in München und Mailand nahezu der gleiche Wind herrschen, denn die Wirkung des vergangenen Tages bätte sicb über Nacht erschöpft, die des neuen hätte noch nicht begonnen; es müfste der Wind der geographischen Breite entsprecben. Man hat aber am Morgen in Müuchen einen Wind von entschieden sudlicher Richtung, in Mailand solchen vou nördlicher, und diese Verschiedenheit kann nur vom Gebirge herrühren. Nachmiltags hat man in München entschieden nördlichen Wind, in Mailand entschieden südlichen; es giebt also auch eigene Tagwinde. Zählt man die einzelnen Projectionen zusammen, so ergiebt sich bei München ein Plus der Südwinde, bei Mailand ein Plus der Nordwinde; es sind daher von den beiden durch das Ge birge hervorgerufenen Winden die Nachtwinde die bedeutenderen, die Nachtwirkung ist die stärkere. Weun von dem Einflusse der Gebirge auf die Luftströmungen die Rede ist, so hat man fast immer nur die aufsteigenden, gegen das Gebirge gebenden im Auge; allein gerade diese siud die unbedeutenderen.

Niment man an, die in München auf eine ungerade Stunde treffende (S-N) Projection sey gleich dem arithmetischen Mittel der beiden angränzenden geraden Stunden, und berechnet man dann das Mittel der Stunden $7^{\mathrm{h}}$ Morgens, $2^{\mathrm{h}}$ und $9^{b}$ Abends, so erbält man $+7,11$, wäbrend das Mittel der Peifsenberger Beobachtungen $+2,89$ ergiebt. Man hat daher auf dem Hohenpeifsenberge weniger Südwinde als in München, und die Ursache, welche an letzterem Orte Südwinde hervorruft, ist an ersterem weniger oder gar nicht thätig. Die von den Bergen kommenden Strömungen sind kalte Winde, die nur in den Thälern binstreichen. Man kann diese Bergwinde von höheren Punkten aus sebr hăufig sehen, denn sie führen jene Nebelströme mit sich, welche 
enge Gebirgsthäler bis zu einer bestimmten Hühe anfüllen, am Morgen am stärksten sind und verschwinden, sobald die aufsteigenden Winde beginnen. Auf Höben wie der Peifsenberg werden solche Ströme kaum kommen, da sie sich im Flachlande zu sehr vertheilen können, und man hat daher in Hohenpeifsenberg einen weiteren Grund zu den bereits oben angeführten, welche bewirken, dafs dort die Windänderuugen kleiner siud als in München. Wären die Alpeu nicht vorhauden, so wäre der Wind in Hohenpeifsenberg mehr südlich, in Müuchen mehr nördlich, als gegenwärtig gefunden wird, und die Projection $(S-N)$ würde daher auf diesem Theile des Festlandes zwischen 7,11 und 2,89 betrageu.

Die beiden Stationen Karlsrube und Manuheim liegen in der Niederung, sie werden daher auch dem Nachtwinde ausgesetzt seyn; aber dieser ist in Karlsruhe etwas südlich, in Mannheim etwas nördlich. Es unufs daher des Nachtwindes wegen der mittlere Werth der Projection (S-N) an erstereın Orte erhöht, an letzterem erniedrigt werden. Diese Werthe sind für Karlsruhe $=7,62$, für Mannhein $=0,58$, und in der Rheinebene ist daher die Richtung des SW.Stromes nach Abzug der Localeinflüsse nahe oder yanz gleich dem auf der bayerischen Hochebene auftretenden.

Der : Mittelwerth der Projectionen $(\mathbf{W}-\mathbf{O})$ aus den Stunden $7^{\mathrm{h}}, 2^{\mathrm{h}}$ und $9^{\mathrm{b}}$ in München und Hohenpeifsenberg ist nachfolgender:

$\begin{array}{lcc} & \text { München } & \text { Hohenpeifsenberg } \\ \text { Winter } & 15,49 & 24,30 \\ \text { Sommer } & 25,31 & 23,44 \\ \text { Jahr } & 18,01 & 20,15\end{array}$

Im Winter giebt es auf dem Peifsenberge mehr Westwinde als in Munchen. Bekanntlich ist der Ostwind der abgelenkte kalte Nordwind, der Westwind dagegen ist ursprünglich warmer Südwind. Löst der Ostwind den Westwind $a b$, so ist, wenn auch nicht immer doch häufig, der Vorgang der, dafs der kalte Ostwind sich unter den Westwind hineinschiebt, wăhrend dieser seinerseits, wenn er herrschend 
wird, sich vou obeu herab geltend macht. Sind daher zwei Stationen in ungleicher Höbe, so hat die untere den östlichen Wind früher und länger; es mufs daher auch bei ihr die Zahl der Projectionen der Ostwinde eine grölsere [oder $(\mathbf{S}-\mathbf{W})$ kleiner] seyn, wie dieses auch der Vergleich von München und Peilsenberg giebt. Im Sommer ist die Temperaturdifferenz der beiderseitigen Winde kleiner '), wefswegen auch das gegenseitige Verdrängen nach der Höhe zu verschwinden scheint, und die Projectionen $(\mathrm{W}-\mathrm{O}$ ) nahe gleich werden, denn die obige Differenz ist vielleicht nur Folge der nicht genau ausführbaren Interpolation.

An den Stationen Mailand, Karlsruhe und Manuheim wirkt das Gebirge zu Gunsten der ostlichen Winde und die Projectionen ( $\mathbf{W}-\mathbf{O}$ ) wüssen daher kleiner seyn als in München, was auch in der That der Fall ist; doch mufs ich gesteben, dafs ich die Differenz zwischen Karlsruhe und Mannheiin kleiner erwartet hätte. Wirkt vielleicht an letzterem Orte auch der Rhein zu Gunsten der Ostwinde?

Berechnet man nach der Lambert'schen Methode die sogenannten mittleren Winde, so ist es unschwer zu bemerken, dafs diese je nach der $W$ ahl der Beobachtungsstunde für ein und denselben Ort sebr verschieden ausfallen können. Berechnet man den mittleren Wind für München aus den einzelnen Stunden, so ergiebt sich:

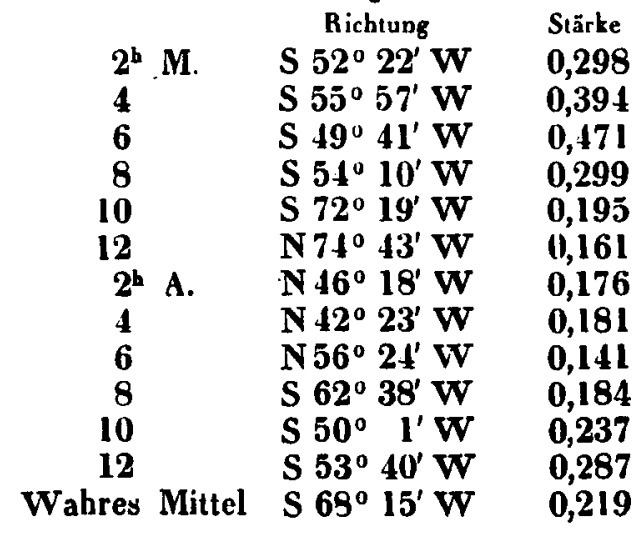

1) Dore, a. a. O. XIV. 
In Mailand und Mannheim wechseln mittlere Windrichtung und Stärke noch webr. Dieser Umstand läfst die Genauigkeit der Bestimmungen von mittlerer Windrichtung in einem sehr zweifelbaften Lichte erscheinen, da es durchaus nicht gleichgültig ist, welche Beobachtungsstunden man hierzu wählt. In München würde sich die letzle Viertelstunde vor $10^{b}$ Morgens eignen, und auch die Counbination $7^{b}, 2^{b}$ und $9^{\text {h }}$ giebt kein ganz schlechtes Resultat; ob aber diese Stunden für andere Orte auch gelten, ist schwer zu bejahen. In Hohenpeifsenberg, wo die Nachtwinde, die von Süden kommen, fehlen, giebt die erwähnte Combination ohne Zweifel einen zu nördlichen Wind.

An anderen Orten kommen wieder andere Localeinflüsse und es dürfte wohl nothwendig seyn zuerst diese Wirkungen näher zu untersuchen, ehe man aus der Vergleichung vieler auf einer gegebenen Ländermasse zerstreuter Orte Schlüsse von Bedeutung auf die gröfseren Luftströmungen ziehen kann. Die Resultate, die man aus der Vergleichung der Beobachtungen mittleren Windes auf dem Festlande bisher erzielt hat, sind daher auch ziemlich zweifelhaften Werthes; man mufs sich für jetzt fast durchaus auf die auf dem hohen Meere gemachten Beobachlungen beschrănken. Das beste Mittel zur Auffindung der Localeinflüsse wären wohl an verschiedenen Orten gleichzeitige Beobachtungen uud Vergleichung der einzelnen Resultate.

Die von den Höhen der Berge herabkommenden Luftströme haben offenbar cine niedrigere Temperatur als die Luft unmittelbar uber der Ebene. Die Bergwinde sind stärker als die von der Ebene hinaufgehenden Landwinde, und jedenfalls sind die eine gegebene Station passirenden derselben nur wenig wärmer als die am Orte selbst. Die Bergwinde erkälten, die Landwinde dagegen erwărmen wenig oder gar nicht, und das Facit mufs daher seyn, dafs das Gebirge die Temperatur des umgebenden Flachlandes erniedrigt. Da das Gebirge vorzugsweise geeignet ist, die kalten Luftströme der Hühe in die wärmeren Tiefen herabzuführen, so mufs auch in der Nähe der Berge der Tem- 
peraturwechsel ein rascherer werden. Es ist bekanut, dais an Orten, die dein Gebirge nahe liegen, die Abende selbst im Hochsommer so kühl sind, dafs man sich leicht Erkältungen zuzieht, wenn man sich in leichlen Kleidern in's Freie setzt. München ist dieses Umstandes wegen bis zum Lächerlichen verschrieen. Es soll dabei der Einflufs der Abkühlung durch Strahlung durchaus nicht geläugnet werden; allein bei einer Höhe von 1600 Fufs kann diese Wirkung doch nicht so bedentend von der der Niederungen verschieden seyn, da noch dazu München durch seine starke Wolkendecke geschützt ist, denn die mittlere Bewölkung beträgt in den Sommermonaten 2,71, 2,64 und 2.57, im Jahresdurchschnitte 2,84 Viertheile des ganzen Himmels') und das kann man eben doch wicht einen ewig beitern Hinmel nennen. Vergleicht man die Temperaturextreme, welche in den einzelnen Monaten an Orten beobachtet werden, die sich in verschiedener Entfernung vom Gebirge befinden, so ergiebt sich, dafs die Schwankungen in der Nähe der Berge gröfser sind. Nachstehende Tabelle giebt den inittleren Werth der Temperaturextreme von Kempten ${ }^{2}$ ), Bogenhausen ${ }^{3}$ ) und Dillingen ${ }^{3}$ ) in Réaumurgraden.

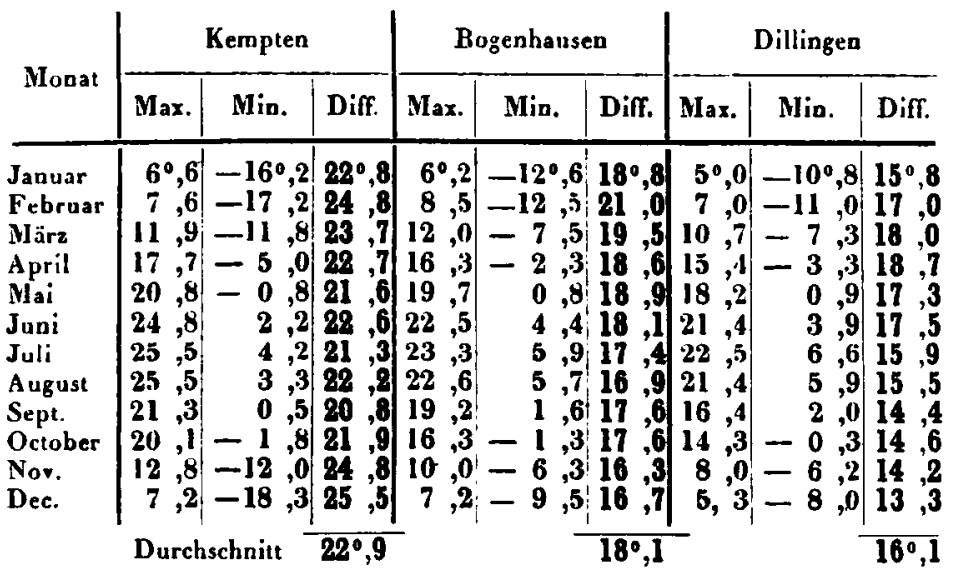

1) Lamont, Monatl. und jährl. Resultate der an der Königl Sternwarte bei München veranstalteten meteorolugischen Untersuchungen. LXX.

2) Achtzehnjährige Beobachtungen des Hrn. Hofapothekers Fuchs, Msc.

3) Lamont, Monatl. und jährl. Resultate etc. 29 Jahre.

4) Achtzehnjährige Beobachiungen des Hrn. Prof. Dr. Pollak, Msc. 
Man sieht hieraus, dafs die 'Temperaturschwankungen un so kleiner werden, je mehr man sich vom Gebirge entfernt.

Wẹn in der Nacht vom Gebirge ausgehende Luftströmungen im Flachlande wehen, so ist nothwendig, dafs in der Höhe der Wind gegen das Gebirge geht, un das dortige Deficit an Luft zu decken. Geht am Tage der Wind unten gegen das Gebirge, so mufs sich die Luft oben ausbreiten und die Folge davon werden Erscheinungen seyn, die man auch anderwärts beobachtet, wo das Aufsteigen der Luft sehr bedeutend ist. An dem Gebirge tindet ein Vorgang statt, der viele Analogie mit jenem der Calmenzone zeigt und in seinem Gefolge mufs eine analoge Wir. kung seyn, die Erhöhung der Regenmenge. Wäre das angränzende Flachland eine $W$ asserfläche, so inüfste das aus derselben aufsteigende $W$ asser sich in der Höhe condensiren und massenhafte Regen würden die Folge seyn. Ist jedoch das Flachland Land, so wird die Verdunstung natürlich nicht so grofs seyn; sie ist aber doch darum nicht zu übersehen, denn sie macht nach v. Sonklar ${ }^{1}$ ) im Dovaugebiete etwa 9 Zehntheile der ganzen Regenmenge aus. Dazu kounmt noch der von v. S ouk la r angeführte Unstand, dafs in der Näbe der Gebirge Luftmassen von verschicdener Wärne viel leichter Gelegenheit geboten wird, sich durcheinander zu mischen als dieses anderwärts der Fall ist. Diese beiden Ursachen wirken auf jedem Abhange eines Gebirges dahin, die Regenınenge zu erböhen; und mit ihuen verbindet sich noch die durch den mechanischen Widerstand der Berge hervorgebrachte Stauung der feuchten Südwestströme auf der Wetterseite des Gebirges, welche Dove nachgewiesen hat, die besonders dazu beiträgt, die Regenmenge auf der Süd-, West - und Südwestseite gröfser zu machen' als die, welche auf dem entgegengesetzten Abhange gefunden wird.

1) Grundzüge einer Hyetographie des österreichischen Kaiserstaates in den Mitıheilungen der Kaiserl. Königl. geogr. Gesellschaft, 1860 S. 217. 